\title{
A theoretical approach to the polymerization of $N$-pyrrolyl ethyl vinyl ether
}

\author{
Mine Yurtsever ${ }^{a}$, Levent Toppare ${ }^{b}, *$ Yusuf Yağcı ${ }^{a}$ \\ ${ }^{a}$ Istanbul Technical University, Chemistry Department, İstanbul, Turkey \\ 'Bilkent University, Chemistry Department, Ankara, Turkey
}

Received 18 July 1997; revised 11 September 1997; accepted 6 October 1997

\begin{abstract}
The oligomerization mechanism of $N$-pyrrolyl ethyl vinyl ether is studied for two different routes of polymerization by using quantum mechanical calculations. Model compounds for oligomerization between monomers and monomer-pyrrole systems are optimized fully via semiempirical methods. By comparing the enthalpy changes of these two processes, it is found that generally the binding of pyrrole groups on the carbon backbone is favoured; however, the self-polymerization is also thermodynamically competitive. These results support the previous experimental evidence. (C) 1998 Elsevier Science B.V.
\end{abstract}

Keywords: Conducting polymers; Semiempirical methods; $N$-pyrrolyl ethyl vinyl ether

\section{Introduction}

The synthesis of conducting polymers has been of considerable interest over the last two decades owing to their unusual applications which involve sensors, photovoltaics and EMI shielding. The main drawback seemed to be their poor mechanical properties and processability. A solution to this problem, to a certain degree, was found in the blending of conducting polymers with common thermoplastic polymers. Several studies have been conducted, either chemically or electrochemically, to produce conducting polymer composites with better physical properties [1-7].

However, as an alternative route for the production of conducting polymeric materials, a new trend is grafting of a conducting chain onto a polymer backbone having a pendant pyrrole or thiophene

\footnotetext{
* Corresponding author.
}

moiety $[8-11]$. The reason for the synthesis of such insulating polymers lies in the fact that they are soluble and can easily be reacted with pyrrole or thiophene in solution through electrochemical [12] or oxidative chemical methods $[13,14]$. We have synthesized poly(2-chloro ethyl vinyl ethers) (Poly A) electrochemically [15] and photochemically. The reaction between Poly A and pyrrolyl potassium yields $N$-pyrrolyl ethyl vinyl ether polymers (Poly B). Poly B can undergo several reactions with and without pyrrole through electrochemical methods. During these studies cyclic voltammetry experiments led us think about the possible ways of reacting Poly B and pyrrole, and possibility of self-reaction for Poly B. In cyclic voltammetry studies, Poly B does not show any electroactivity, whereas pyrrole in the presence of Poly B yields a different electroactivity behaviour than is the case if it were alone in the electrolyte solution. This may be an indication of a reaction 
172

M. Yurtsever et al./Journal of Molecular Structure (Theochem) 430 (1998) 171-176<smiles>C=COCCCl</smiles>

$$
\stackrel{\text { hv }}{\longrightarrow} \rightarrow
$$

Poly A.<smiles>CCCOCCCl</smiles>

Poly A

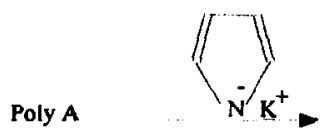<smiles>[CH]COC[CH2]</smiles>

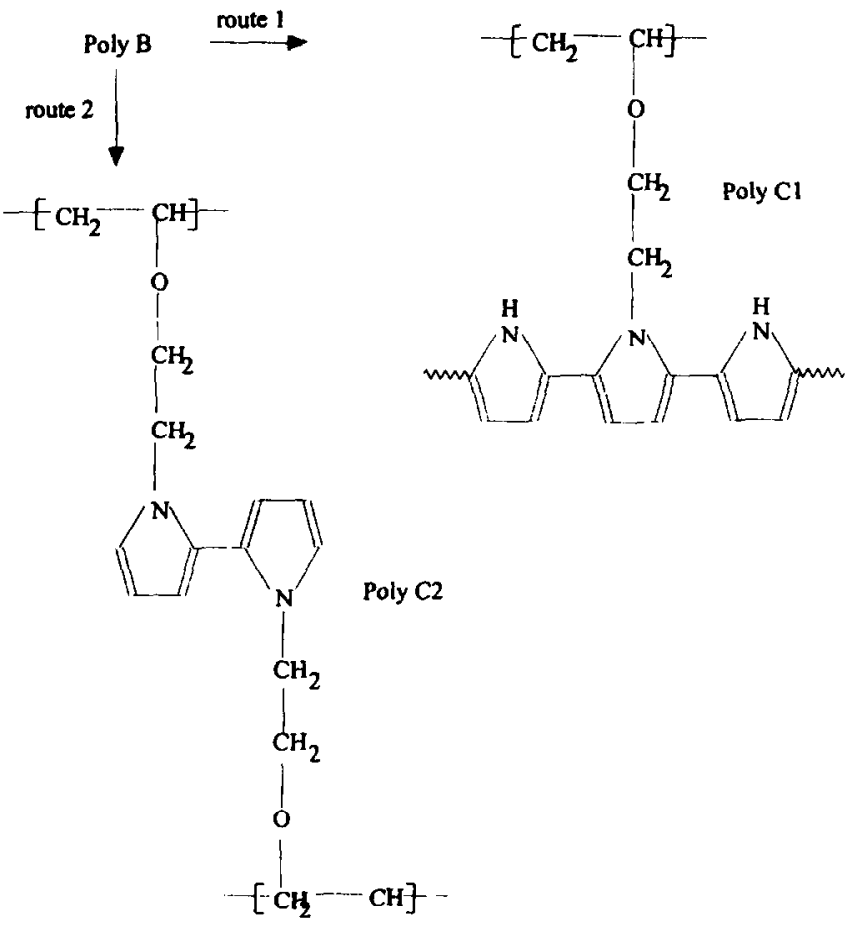


<smiles>CCOCCN1CCOC1</smiles>

Molecule 1<smiles>CCCOCCN1CCOC1</smiles>

Molecule 2<smiles>CC(C)(C)OCCN1CCOC1</smiles>

Molecule 5

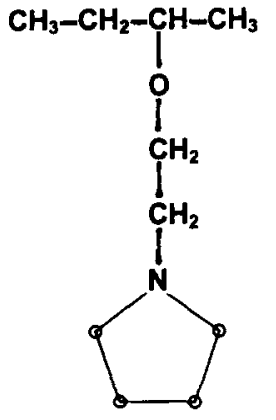

Molecule 3

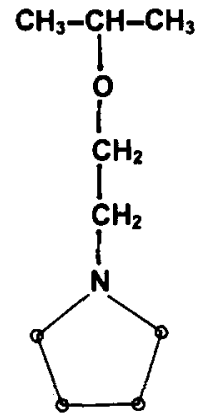

Molecule 4

Fig. 1. Model monomers (continued).

between pyrrole and the pyrrole moiety of Poly B. $\Lambda$ fter several runs (ca. 20), however, the voltammograms become exactly the one revealed by pyrrole taken in the same medium in the absence of Poly B. This is most probably a consequence of the presence of long pyrrole chains on the backbone, and the polymer (Poly $\mathrm{C} 1$ ) behaves as polypyrrole. The lack of electroactivity in the cyclic voltammogram of Poly $B$ in the absence of pyrrole led us run preparative constant potential electrolyses where only Poly B exists in the medium. Details of the experimental work can be found elsewhere [16]. The resultant polymer (Poly $\mathrm{C} 2$ ), as coated on the anode, was not only non-conducting but also very low in yield $(2 \%)$.

The following questions were challenging from the theoretical point of view. When the pyrrole is absent, how feasible is the self-polymerization of Poly B to Poly C2 (route 2)? When the pyrrole is present in the medium, which polymerization is more probable? How do these two reactions compete? We have previously carried out semiempirical quantummechanical calculations on polymerization mechanisms of five-membered rings [17,18] and obtained reasonable insight to the relative probabilities of different mechanisms. In particular, we were able to obtain information on the various growth possibilities leading to braching of chains. In a similar fashion, we proceed to apply semiempirical quantum-mechanical machinery to answer the questions mentioned above. In this work we have used the AM1 option of MOPAC 6.0 [19] as well as the molecular modelling program MOLCAD [20] running on Silicon Graphics workstations. 


\section{Results and discussion}

Since calculations involving of the actual polymer chains are still not possible even for semiempirical approaches (except for highly symmetrical forms), we begin by modelling Poly B by five different monomers with varying numbers of carbon atoms on the backbone and using them as the starting material for oligomerization reactions (Fig. 1). In Table 1 we present the formation enthalpies of monomers as well as that of pyrrole. We then proceed to calculate optimized structures of the products obtained from two different reactions, i.e., the addition of pyrrole to the pyrrole unit of Poly B (route 1)

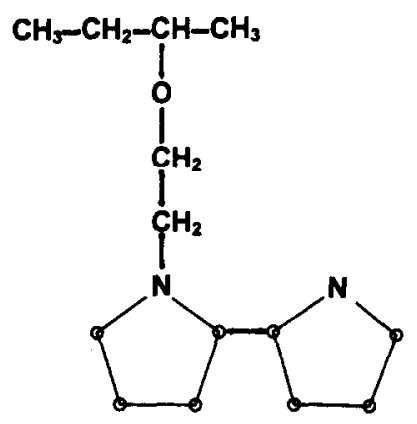

\section{Dimer Poly C1}

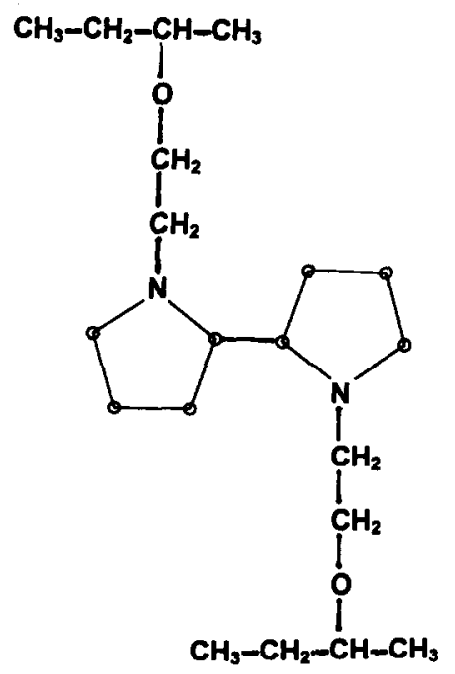

Table 1

Enthalpy of formation for the molecules involved in oligomerizations

\begin{tabular}{ll}
\hline Molecule & $\Delta_{\mathrm{f}} \mathrm{H}\left(\mathrm{kcal} \mathrm{mol}^{-1}\right)$ \\
\hline 1 & -8.17 \\
2 & -14.40 \\
3 & -16.97 \\
4 & -11.60 \\
5 & -11.79 \\
Pyrrole & 39.89 \\
$\mathrm{H}_{2}$ & -5.18 \\
\hline
\end{tabular}

Fig. 2. Dimers of Poly $\mathrm{Cl}$ and Poly C2 (molecule 3). 
Table 2

Reaction enthalpies (in $\mathrm{kcal} \mathrm{mol}^{-1}$ ) for two routes of dimerization

\begin{tabular}{lcc}
\hline Molecule & Poly C1 & Poly C2 \\
\hline 1 & -1.63 & -0.62 \\
2 & -1.68 & -1.02 \\
3 & -3.52 & -2.22 \\
4 & -4.46 & -1.05 \\
5 & -5.39 & -4.27 \\
\hline
\end{tabular}

and self-addition of Poly B (route 2) (Fig. 2). The model reactions involving up to five monomers are studied for both routes. In order to find out relative likelihoods of these processes we need to compare the reaction enthalpies rather than the individual enthalpies of formation, since the two reactions to be studied involve different numbers of atoms. The results for the dimerization reactions are given in Table 2. The linkages between pyrrole rings are via $\alpha$ carbons as the semiempirical calculations had suggested [18].

It seems that the resulting products become more stable as the carbon backbone is increased, implying that indeed the addition of the pyrrole ring is a favourable process for Poly $\mathrm{B}$. However, the most stable dimer has a tertiary carbon connected to oxygen. Extension of the carbon skcleton in three directions creates branching, which makes the polymerizations difficult owing to steric interactions. The comparison of reaction enthalpies for the two processes indicates that the addition of pyrrole ring (Poly C1) is always favourable to the extent of $1 \mathrm{kcal} \mathrm{mol}^{-1}$ over the selfdimerizations. One exception is monomer 4 , where this difference is around $3.5 \mathrm{kcal} \mathrm{mol}^{-1}$. which may be due to the symmetrical form of the carbon backbone. For growing into larger units we have used the monomers 3 and 4 . The choice of these monomers is because of their symmetrical forms for the carbon backbone, so a better representation of the long chains can be achieved. Trimers are formed either by the

Table 3

Reaction enthalpies (in $\mathrm{kcal} \mathrm{mol}^{-1}$ ) for oligomerization with three and four units

\begin{tabular}{|c|c|c|c|c|}
\hline \multirow[t]{2}{*}{ Molecule } & \multicolumn{2}{|l|}{ Trimer } & \multicolumn{2}{|l|}{ Tetramer } \\
\hline & Poly $\mathrm{Cl}$ & Poly C2 & Poly Cl & Poly C2 \\
\hline 3 & -4.57 & -1.41 & -3.01 & -2.11 \\
\hline 4 & -4.11 & -0.97 & -3.03 & -1.73 \\
\hline
\end{tabular}
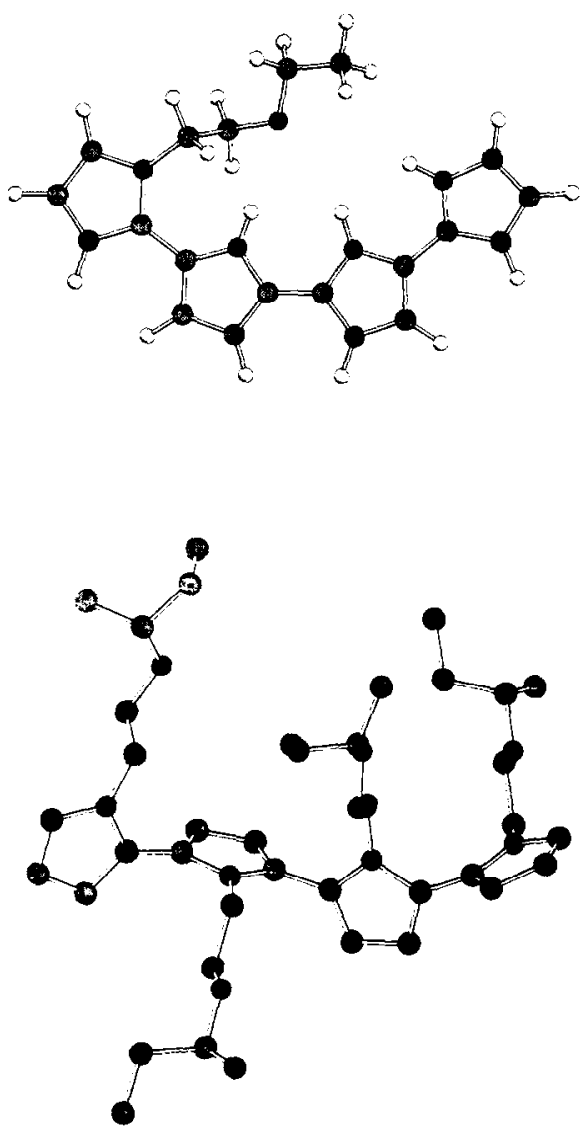

Fig. 3. Examples of three-dimensional structures of tetramers of Poly Cl and Poly C2.

addition of two pyrrole rings to Poly B or by combining three monomers, and finally similar structures for tetramers are generated for both routes. In Table 3 we present results for these oligomers.

Again, similar to the results for the dimerization processes, the reaction enthalpies are lower for the Poly $\mathrm{Cl}$ route by at least $1 \mathrm{kcal} \mathrm{mol}^{-1}$ than for the self-attachment of monomer units. The threedimensional structures for the oligomers of Poly C2 (Fig. 3) also suggest that chains involving a large number of monomers are not easy to grow because of steric interactions.

Finally, we should like to conclude that, in the presence of pyrrole, the self-polymerization of Poly $\mathrm{B}$ is in competition with pyrrole addition. Both experimental results from the electrochemistry of 
the processes and quantum-mechanical calculations of the reaction enthalpies, as well as the optimum structures, reveal that Poly $\mathrm{Cl}$ dominates the formation of Poly $\mathrm{C} 2$.

\section{References}

[1] H.L. Wang, L. Toppare, J.E. Fernandez, Macromolecules 23 (1990) 1053

[2] U. Geissler, M.L. Hallensleben, L. Toppare, Adv. Mater. 104 (1991) 3.

[3] E. Yurtsever, L. Toppare, M. Hallensleben, J. Mol. Struct. (Theochem) 280 (1993) 1.

[4] S. Doğan, U. Akbulut, L. Toppare, Synth. Met. 53 (1992) 29.

[5] F. Selampınar, U. Akbulut, T. Yalçın, SSüzeŗ., , L. Toppare, Synth. Met. 62 (1994) 201.

[6] F. Selampınar, L. Toppare, U. Akbulut, T. Yalçın, S Süzeŗ., Synth. Met. 68 (1995) 109.

[7] N. Balci, L. Toppare, E. Bayramlı, Composites 26 (1995) 1.
[8] D. Stanke, M.L. Hallensleben, L. Toppare, Synth. Met. 73 (1995) 261

[9] D. Stanke, M.L. Hallensleben, L. Toppare, Macromol. Chem. Phys. 196 (1995) 1697.

[10] D. Stanke, M.L. Hallensleben, L. Toppare, Synth. Met. 72 (1995) 167

[11] D. Stanke, M.L. Hallensleben, L. Toppare, Synth. Met. 72 (1995) 89.

[12] N. Balcı, U. Akbulut, L. Toppare, D. Stanke, M.L. Hallensleben, Mater. Sci. Bull., 32 (1997) 1449.

[13] D. Stanke, M.L. Hallensleben, L. Toppare, Synth. Met. 72 (1995) 95.

[14] S. Bahçeci, L. Toppare, E. Yurtsever, Synth. Met. 81 (1996) 5.

[15] E. Kalaycıoğlu, L. Toppare, Y. Yağcı, J. Macromol. Sci., Pure Appl. Chem., 89 (1997) 111.

[16] F. Selampınar, U. Akbulut, E. Yıldız, A. Güngör, L. Toppare, Synth. Met., in press.

[17] G. Bağcı, L. Toppare, E. Yurtsever, Synth. Met. 78 (1996) 19.

[18] P.S. Sargin, L. Toppare, E. Yurtsever, Polymer 37 (1996) 7.

[19] MOPAC version 5.00, Quantum Chem. Program Exch., 455.

[20] J. Brickmann, et al., MOLCAD, Molecular Modelling Program, Technische Hochschule Darmstadt. 